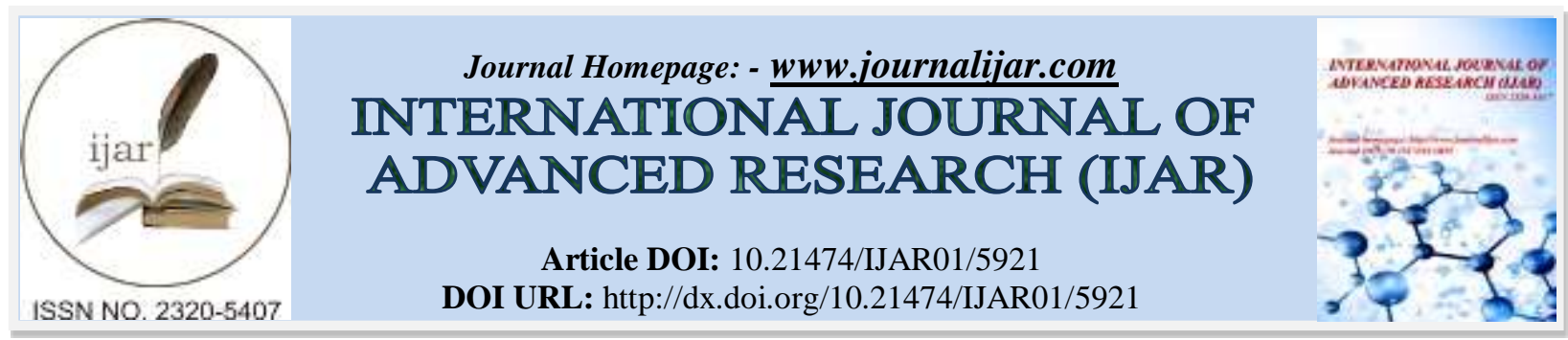

RESEARCH ARTICLE

\title{
CORRELATION OF LIPID PROFILE WITH CAROTID INTIMA MEDIA THICKNESS IN NEWLY DIAGNOSED CORONARY ARTERY DISEASE PATIENTS.
}

1. Department of Medicine, Yenepoya Medical College Hospital.

2. Asst. Professor, Department of Medicine, Yenepoya Medical College Hospital.

\section{Manuscript Info}

Manuscript History

Received: 20 September 2017

Final Accepted: 22 October 2017

Published: November 2017

\section{Abstract}

Copy Right, IJAR, 2017,. All rights reserved.

Project Summary:-

- Coronary Artery Disease (CAD) is a major health problem with considerable morbidity and mortality and has been long eluding our efforts for an effective control. Knowledge of risk factors with early indicators will help to prevent the occurrence of CAD.

- Carotid-wall intima-media thickness (CIMT) is a surrogate measure of atherosclerosis associated with Cardiovascular risk factors and outcomes.

- The intima-media thickness is the distance from the lumen-intima interface to the media-adventitia interface of the artery wall, as measured on noninvasively acquired ultrasonographic images of carotid arteries.

- Increased intima-media thickness of the common carotid artery represents a form of atherosclerosis that is manifested as diffuse arterial-wall thickening whereas increased intima-media thickness of the proximal internal carotid artery is a surrogate for focal atherosclerotic plaque.

- The mean intima-media thickness of the common carotid artery is a more reproducible measure than the intima-media thickness of the internal carotid artery and is believed to be better suited for cardiovascular risk assessment and intervention studies.

- There is overwhelming amount of evidence relating to high levels of serum, Total and LDL Cholesterol and low levels of cholesterol with coronary atherosclerosis, the relation between serum lipids, lipoproteins Coronary atherosclerosis is less clear.

\section{Objectives Of Study:-}

Thus this study is intended to correlate lipid profile Total cholesterol with LDL:HDL ratio and Triglycerides CIMT thickness in newly diagnosed coronary artery disease patient.

\section{Review Of Literature:-}

- Caie Yang et al did a study on the correlation between serum lipid profile with carotid intima media thickness and plaque in 2014 and concluded that the serum LDL-C/HDL-C ratio represents as an independent index associate with increased carotid IMT and LDL-C combined with HDL-C levels may be useful markers for predicting the presence of carotid plaque in the Chinese general population. 
- Samia Perwaiz et al conducted a study in 2008 on the variations in Carotid intima media thickness in familial Hypercholesterolemia patients and its use as predictive marker for premature cardiovascular disease. In this study CIMT was found to be significantly increased in familial Hypercholesterolemia and it correlated with raised LDL-cholesterol. Both were predictors of premature cardiovascular disease.

- Debalina Sengupta et al conducted a study in 2014 on correlation between lipid profile and carotid intima media thickness in cerebral ischaemia and concluded that the altered lipid profile is associated with cerebral ischaemia by increasing carotid intima media thickness (IMT) .

- Preetha et al conducted a study on lipid profile and carotid intima thickness in diabetic and non diabetic ischaemic stroke patients in 2017 and concluded that the lipid parameters including TC, TG,LDL and VLDL were significantly raised in diabetic stroke patients and had positive correlation with the risk of stroke.

\section{Materials \& Methods:-}

$\begin{array}{lll}\text { Study Design } & : & \text { Observational Study } \\ \text { Study Setting } & : & \text { Yenepoya Medical College Hospital } \\ \text { Study Duration } & : & \text { 20th Oct 2017 - 20th Nov 2017 } \\ \text { Source of Data } & : & \text { Patients admitted in YMCH } \\ \text { Sample Size } & : & 50 \text { cases which meet inclusion criteria }\end{array}$

* The present study will be carried out after obtaining the Ethical committee clearance. Relevant clinical data (demographic- age, sex, place, occupation) including history will be obtained from patients and detailed clinical examination will be performed.

* The risk and complications of coronary angiogram will be explained to the patient like reperfusion arrhythmias, artery rupture, bleeding, infections.

- Subsequently patient with established Coronary Artery Disease had undergone CAROTID DOPPLER to estimate the carotid intima media thickness.

* Patient were examined in supine position with head tilted backward. After the carotid arteries will be located by B mode ultrasonography Equipped with $7.5 \mathrm{mhz}$ linear array transducer. The probe was rotated right angle to obtain and record a longitudinal image of anterior and posterior walls. The measurement can vary from one observer to another.

* The maximum IMT will be measured at the near and far wall of common carotid artery, the bifurcation and the internal carotid arteries and were expressed as a mean aggregated value.

* Lipid profile after 12 hours of overnight fasting will be done and total cholesterol will be measured by pap method, Triglycerides by Pap method LDL:HDL ratio (HDL by PEG Precipitation method and LDL by Friedewald's formula).

\section{Inclusion Criteria:-}

* Newly diagnosed coronary artery disease patients by coronary angiogram

* Age between 40-70 age group

\section{Exclusion Criteria:-}

* Patient with H/ CAD, Stroke

* Patients already on statins

* Other chronic inflammatory conditions, chronic kidney disease

\section{Statistical Analysis:-}

* Chi-Square test

* Student T- tests

Results:-

\begin{tabular}{|l|l|l|l|l|l|l|l|}
\hline \multicolumn{2}{|l|}{ Correlations } & AGE & CIMT & $\begin{array}{l}\text { Total } \\
\text { cholesterol }\end{array}$ & Triglycerides & HDL & LDL \\
\hline \multirow{2}{*}{ AGE } & $\begin{array}{l}\text { Pearson } \\
\text { Correlation }\end{array}$ & 1 & .176 & .156 & .186 & .037 & .126 \\
\cline { 2 - 8 } & Sig. (2-tailed) & & .223 & .279 & .196 & .799 & .382 \\
\hline
\end{tabular}




\begin{tabular}{|c|c|c|c|c|c|c|c|}
\hline & $\mathrm{N}$ & 50 & 50 & 50 & 50 & 50 & 50 \\
\hline \multirow[t]{3}{*}{ CIMT } & $\begin{array}{l}\text { Pearson } \\
\text { Correlation }\end{array}$ & .176 & 1 & $.868^{* * *}$ & $.4333^{* * *}$ & .043 & $.909^{* * *}$ \\
\hline & Sig. (2-tailed) & .223 & & .000 & .002 & .768 & .000 \\
\hline & $\mathrm{N}$ & 50 & 50 & 50 & 50 & 50 & 50 \\
\hline \multirow[t]{3}{*}{$\begin{array}{l}\text { Total } \\
\text { cholesterol }\end{array}$} & $\begin{array}{l}\text { Pearson } \\
\text { Correlation }\end{array}$ & .156 & $.868^{* * *}$ & 1 & $.294^{*}$ & .223 & $.937^{* *}$ \\
\hline & Sig. (2-tailed) & .279 & .000 & & .038 & .120 & .000 \\
\hline & $\mathrm{N}$ & 50 & 50 & 50 & 50 & 50 & 50 \\
\hline \multirow[t]{3}{*}{ Triglycerides } & $\begin{array}{l}\text { Pearson } \\
\text { Correlation }\end{array}$ & .186 & $.433^{* * *}$ & $.294^{*}$ & 1 & -.095 & $.389^{* * *}$ \\
\hline & Sig. (2-tailed) & .196 & .002 & .038 & & .510 & .005 \\
\hline & $\mathrm{N}$ & 50 & 50 & $\mathbf{5 0}$ & 50 & 50 & 50 \\
\hline \multirow[t]{3}{*}{ HDL } & $\begin{array}{l}\text { Pearson } \\
\text { Correlation }\end{array}$ & .037 & .043 & .223 & -.095 & 1 & .040 \\
\hline & Sig. (2-tailed) & .799 & .768 & .120 & .510 & & .783 \\
\hline & $\mathrm{N}$ & 50 & 50 & 50 & 50 & 50 & 50 \\
\hline \multirow[t]{3}{*}{$\begin{array}{l}\mathrm{LDL} \\
\end{array}$} & $\begin{array}{l}\text { Pearson } \\
\text { Correlation }\end{array}$ & .126 & $.909^{* *}$ & $.937^{* *}$ & $.389^{* * *}$ & .040 & 1 \\
\hline & Sig. (2-tailed) & .382 & .000 & .000 & .005 & .783 & \\
\hline & $\mathrm{N}$ & 50 & 50 & 50 & 50 & 50 & 50 \\
\hline
\end{tabular}

\section{Correlation interpretation:}

0 to .3 - weak positive correlation

; $\quad 0$ to -.3 -weak negative correlation

.4 to .7 - moderate positive correlation ;

$\mathbf{- 0 . 4}$ to $\mathbf{- 0 . 7}$ - moderate negative correlation

$>.7 \quad$ - strong positive correlation

; $\quad>-0.7 \quad$ - strong negative correlation

Linear correlation between carotid Intima media thickness with lipid parameters and clinical characteristics were evaluated by Pearson correlation or Spearman rank correlation as appropriate and student $\mathrm{T}$ test was applied to find comparison of lipid profiles with risk factors and normal groups.

There was signification correlation between carotid intima media thickness with LDL ,total cholesterol, tryglycerides $(\mathrm{p}<0.05)$

No significant correlation was seen between Carotid intima media thickness with HDL, Age.

The mean Age in Diabetic group was 53.67, Diabetic with Hypertension group was 52.26, Hypertensive group was 57.5 and No risk factor group was 52.3

Comparison of lipid profiles between the risk factors

\begin{tabular}{|c|c|c|c|c|c|c|}
\hline & & $\mathrm{N}$ & Mean & Std. Deviation & Minimum & Maximum \\
\hline \multirow[t]{4}{*}{ AGE } & DM & 9 & 53.67 & 8.718 & 45 & 73 \\
\hline & DM/HTN & 23 & 52.26 & 6.870 & 40 & 69 \\
\hline & HTN & 6 & 57.50 & 4.370 & 50 & 62 \\
\hline & NORMAL & 12 & 52.33 & 6.597 & 40 & 63 \\
\hline \multirow[t]{4}{*}{ CIMT } & DM & 9 & 1.022 & .1856 & .8 & 1.3 \\
\hline & DM/HTN & 23 & 1.003 & .2164 & .7 & 1.4 \\
\hline & HTN & 6 & .950 & .1517 & .8 & 1.2 \\
\hline & NORMAL & 12 & .975 & .2137 & .7 & 1.4 \\
\hline \multirow[t]{2}{*}{ Total cholesterol } & DM & 9 & 180.00 & 41.533 & 130 & 250 \\
\hline & DM/HTN & 23 & 196.96 & 65.466 & 120 & 350 \\
\hline
\end{tabular}




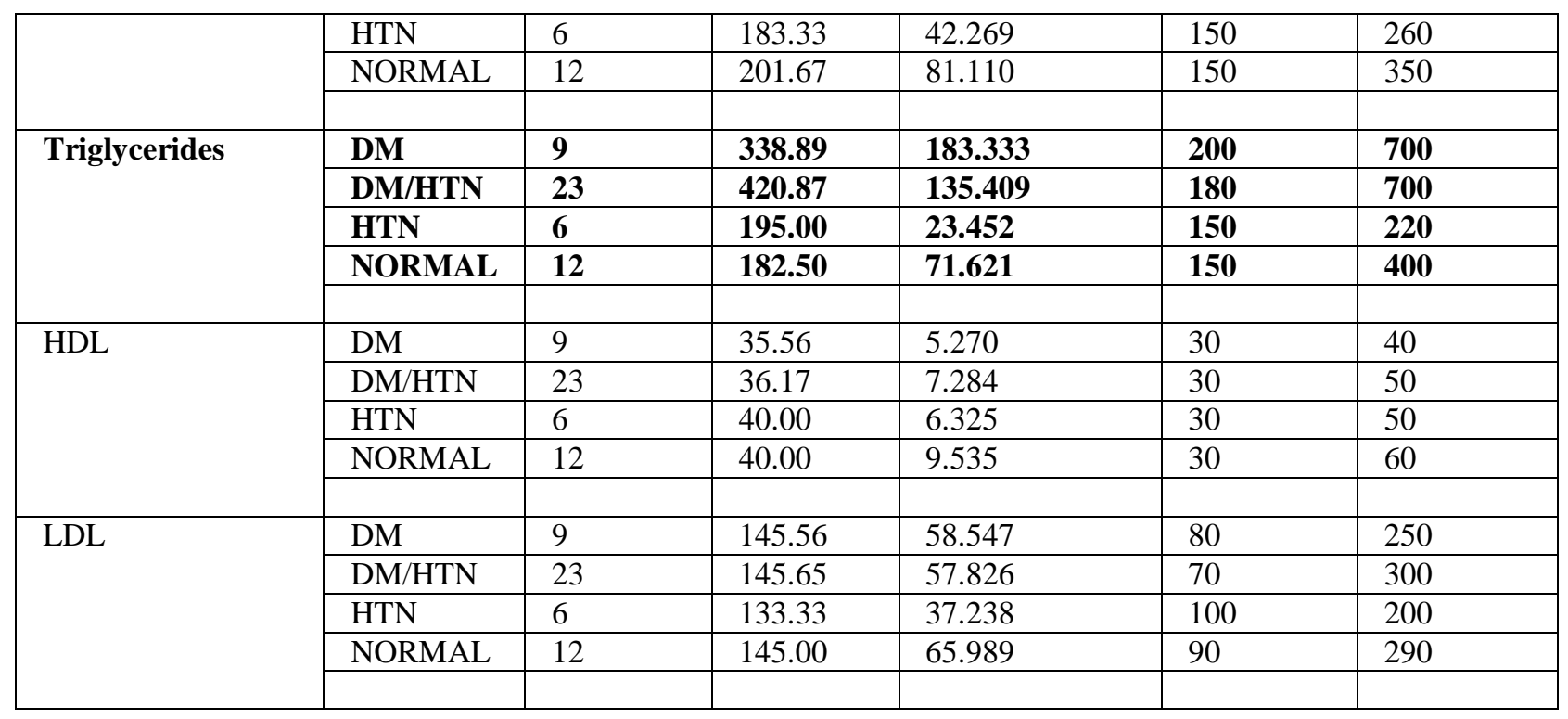

\begin{tabular}{|c|c|c|c|c|c|c|}
\hline \multicolumn{7}{|l|}{ ANOVA } \\
\hline & & $\begin{array}{ll}\text { Sum } & \text { of } \\
\text { Squares }\end{array}$ & df & Mean Square & $\mathrm{F}$ & Sig. \\
\hline \multirow[t]{3}{*}{ AGE } & Between Groups & 142.119 & 3 & 47.373 & .981 & .410 \\
\hline & Within Groups & 2220.601 & 46 & 48.274 & & \\
\hline & Total & 2362.720 & 49 & & & \\
\hline \multirow[t]{3}{*}{ CIMT } & Between Groups & .025 & 3 & .008 & .201 & .895 \\
\hline & Within Groups & 1.923 & 46 & .042 & & \\
\hline & Total & 1.948 & 49 & & & \\
\hline \multirow[t]{3}{*}{ Total cholesterol } & Between Groups & 3335.043 & 3 & 1111.681 & .270 & .847 \\
\hline & Within Groups & 189386.957 & 46 & 4117.108 & & \\
\hline & Total & 192722.000 & 49 & & & \\
\hline \multirow[t]{3}{*}{ Triglycerides } & $\begin{array}{l}\text { Between } \\
\text { Groups }\end{array}$ & 557691.502 & 3 & 185897.167 & 11.691 & .000 \\
\hline & Within Groups & 7314446.498 & 46 & 15901.011 & & \\
\hline & Total & 1289138.000 & 49 & & & \\
\hline \multirow[t]{3}{*}{ HDL } & Between Groups & 186.793 & 3 & 62.264 & 1.106 & .356 \\
\hline & Within Groups & 2589.527 & 46 & 56.294 & & \\
\hline & Total & 2776.320 & 49 & & & \\
\hline \multirow[t]{3}{*}{ LDL } & Between Groups & 779.227 & 3 & 259.742 & .077 & .972 \\
\hline & Within Groups & 155820.773 & 46 & 3387.408 & & \\
\hline & Total & 156600.000 & 49 & & & \\
\hline
\end{tabular}

Comparison of lipid profiles between the presence/ absence of risk factors:

\begin{tabular}{|c|c|c|c|c|c|c|c|c|}
\hline \multicolumn{9}{|c|}{ Group Statistics } \\
\hline & $\begin{array}{l}\text { RF } \\
\text { Y_N }\end{array}$ & $\mathrm{N}$ & Mean & $\begin{array}{l}\text { Std. } \\
\text { Deviation }\end{array}$ & $\begin{array}{l}\text { Mean } \\
\text { difference }\end{array}$ & $\mathrm{T}$ & $\mathrm{df}$ & p-value \\
\hline \multirow{2}{*}{ AGE } & 0 & 12 & 52.33 & 6.597 & -1.088 & -.469 & 48 & .641 \\
\hline & 1 & 38 & 53.42 & 7.115 & & & & \\
\hline \multirow{2}{*}{ CIMT } & 0 & 12 & .975 & .2137 & -.0245 & -.367 & 48 & .715 \\
\hline & 1 & 38 & .999 & .1973 & & & & \\
\hline \multirow{2}{*}{$\begin{array}{l}\text { Total } \\
\text { cholesterol }\end{array}$} & 0 & 12 & 201.67 & 81.110 & 10.877 & .520 & 48 & .606 \\
\hline & 1 & 38 & 190.79 & 56.777 & & & & \\
\hline \multirow[t]{2}{*}{ Triglycerides } & 0 & 12 & 182.50 & 71.621 & -183.289 & -3.868 & 48 & .000 \\
\hline & 1 & 38 & 365.79 & 158.227 & & & & \\
\hline
\end{tabular}




\begin{tabular}{|l|l|l|l|l|l|l|l|l|}
\hline \multirow{2}{*}{ HDL } & 0 & 12 & 40.00 & 9.535 & 3.368 & 1.363 & 48 & .179 \\
\cline { 2 - 9 } & 1 & 38 & 36.63 & 6.724 & & & & \\
\hline \multirow{2}{*}{ LDL } & 0 & 12 & 145.00 & 65.989 & 1.316 & .070 & 48 & .945 \\
\cline { 2 - 9 } & 1 & 38 & 143.68 & 54.198 & & & & \\
\hline
\end{tabular}

\begin{tabular}{|c|c|c|c|c|c|}
\hline \multicolumn{6}{|c|}{ Independent Samples Test } \\
\hline & \multicolumn{5}{|c|}{ T-test for Equality of Means } \\
\hline & $\mathrm{T}$ & Df & p-value & $\begin{array}{l}\text { Mean } \\
\text { Difference }\end{array}$ & $\begin{array}{l}\text { Std. Error } \\
\text { Difference }\end{array}$ \\
\hline AGE & -.469 & 48 & 641 & -1.088 & 2.318 \\
\hline CIMT & -.367 & 48 & .715 & -.0245 & .0666 \\
\hline Total cholesterol & .520 & 48 & .606 & 10.877 & 20.923 \\
\hline Triglycerides & -3.868 & 48 & .000 & -183.289 & 47.381 \\
\hline HDL & 1.363 & 48 & 179 & 3.368 & 2.471 \\
\hline LDL & .070 & 48 & .945 & 1.316 & 18.913 \\
\hline
\end{tabular}

When Student T test was applied to find comparison between lipid profile with presence /Absence of risk factors it is found that cases with risk factors had a higher triglycerides level compared to cases with no risk factors

\section{Discussion:-}

Although preventable atherosclerotic cardiovascular disease remains a leading global cause of death and disability. Causal risk factors for cardiovascular disease constitute important therapeutic targets, but their usefulness as predictors for the disease developing is limited.

Therefore, the only effective approach to restrict the health burden is to prevent the diseases from developing at the earliest possible stage of atherosclerosis.

Thus, screening for biomarkers for detecting early stage asymptomatic vascular atherosclerosis are needed.

Carotid intima-media thickness is a valuable research tool that can be used as surrogate end points in clinical trials $^{1}$

This study was conducted on 50 patients at Yenepoya medical college, Mangalore.

All the patients were newly diagnosed coronary artery disease. CIMT was measured by B mode ultrasonagraphy and fasting lipid profile were measured.

The mean age in Diabetic ( $\mathrm{n}-9$ 53.67), Diabetic with hypertensive group ( $\mathrm{n}=23$ 52.26), Hypertensive group( $\mathrm{n}=6$ 57.50) and no risk factor group ( $\mathrm{n}=12 \mathrm{p} 52.33)$.

Mean carotid intima media in diabetic group (1.02 mm),Diabetic with Hypertensive group $(1.003 \mathrm{~mm})$,Hypertensive group were $(0.95 \mathrm{~mm})$ and no risk factor group $(0.975 \mathrm{~mm})$

This study showed there was positive correlation of carotid intima media thickness with LDL, Total cholesterol and triglycerides.

Similar studies were done by Sengaupta Debalina et $\mathrm{l}^{2}$ where CIMT had a positive correlation between LDL, Total cholesterol and Triglycerides. 
Study also showed that patients with risk factors had higher triglycerides compared to patients with no-risk factors similar to study conducted by Khan SP et al ${ }^{3}$.

Age, Gender did not show any significant correlation. In clinical practice, measurement of CIMT greater than $1.0 \mathrm{~mm}$ in the common carotid artery or presence of plaque may support an early intervention therapy. CIMT can also be used to assess safety and efficacy of cardiovascular medicines . ${ }^{4}$

\section{Conclusion:-}

Carotid intima media thickness measured through Mode ultrasonagraphy was found to be significantly correlated with lipid profile. Thus, it can be used to assess the risk of cardiovascular diseases as it also correlated to increase LDL-CHOLESTEROL and total cholesterol and thus both are predictive markers for premature cardiovascular diseases.

\section{References:-}

1. Silesen H, Falk E: Why not screen for subclinical atherosclerosis? Lancet 2011, 378(9792):645-646.

2. Sengupta D, Bardhan J \& Mahapatra AB Correlation between Lipid Profile and carotid intima media thickness in cerebral ischaemia. Indian J Physiol Pharmacol 2014;58(4):354-364: ; 2013.

3. Khan SP, Ahmed KZ \& Yaqub Z Carotid Intima-Media Thickness Correlation with Lipid Profile in Patients With Familial Hypercholesterolemia Versus Controls, Journal of the College of Physicians and Surgeon Pakistan, Vol 21(1):30-33 2011

4. Espeland MA ,O 'Leary DH,Terry JG ,Morgan $\mathrm{T}$ et al Carotid Intima -media thickness as a surrogate for cardiovascular events in trials of HMG-CoA reductase inhibitor. Curr Control Trials,Cardiovasc Med 2005;6:3

5. Yang $\mathrm{C}$, Sun $\mathrm{Z} \& \mathrm{Li}$ Y The correlation between serum lipid profile with carotid intima-media thickness and plaque, China : BMC Cardiovascular disorders ; 2014.

6. Pillai R, Tiwari D \& Jatav OP Lipid Profile and carotid artery Intima-Media thickness in diabetic and non diabetic ischaemic stroke, International Journal of Advances in Medicine 2017 April;4(2):471-475: ; 2017.

7. Lim S, Despres JP, Koh KK: Prevention of atherosclerosis in overweight/obese patients. - In need of novel multi-targeted approaches. Circ J. 2011, 75 (5): 1019-1027. 10.1253/circj.CJ-10-1240.

8. Nakamura H, Arakawa K, Itakura H, Kitabatake A, Goto Y, Toyota T, Nakaya N, Nishimoto S, Muranaka M, Yamamoto A, Mizuno K, Ohashi Y, MEGA Study Group: Primary prevention of cardiovascular disease with pravastatin in Japan (MEGA Study): a prospective randomised controlled trial. Lancet. 2006, 368 (9542): 11551163. 10.1016/S0140-6736(06)69472-5.

9. Cannon CP, Braunwald E, McCabe CH, Rader DJ, Rouleau JL, Belder R, Joyal SV, Hill KA, Pfeffer MA, Skene AM, Pravastatin or Atorvastatin Evaluation and Infection Therapy-Thrombolysis in Myocardial Infarction 22 Investigators: Intensive versus moderate lipid lowering with statins after acute coronary syndromes. N Engl J Med. 2004, 350 (15): 1495-1504. 10.1056/NEJMoa040583.

10. Miller NE, Thelle DS, Forde OH, Mjos OD: The Tromso heart-study. High-density lipoprotein and coronary heart-disease: a prospective case-control study. Lancet. 1977, 1 (8019): 965-968.

11. Expert Panel on Detection E, Treatment of High Blood Cholesterol in A: Executive summary of the third report of The National Cholesterol Education Program (NCEP) expert panel on detection, evaluation, and treatment of high blood cholesterol in adults (Adult Treatment Panel III). JAMA. 2001, 285 (19): 2486-2497. 10.1001/jama.285.19.2486.

12. Cui Y, Blumenthal RS, Flaws JA, Whiteman MK, Langenberg P, Bachorik PS, Bush TL: Non-high-density lipoprotein cholesterol level as a predictor of cardiovascular disease mortality. Arch Intern Med. 2001, 161 (11): 1413-1419. 10.1001/archinte.161.11.1413.

13. Bittner V, Hardison R, Kelsey SF, Weiner BH, Jacobs AK, Sopko G, Bypass Angioplasty Revascularization I: Non-high-density lipoprotein cholesterol levels predict five-year outcome in the Bypass Angioplasty $\begin{array}{llllll}\text { Revascularization Investigation } & \text { (BARI). Circulation. 2002, } 106 & \text { (20): 2537-2542. }\end{array}$ 10.1161/01.CIR.0000038496.57570.06.

14. Xydakis AM, Ballantyne CM: Role of non-high-density lipoprotein cholesterol in prevention of cardiovascular disease: updated evidence from clinical trials. Curr Opin Cardiol. 2003, 18 (6): 503-509. 10.1097/00001573200311000-00012.

15. Ridker PM, Rifai N, Cook NR, Bradwin G, Buring JE: Non-HDL cholesterol, apolipoproteins A-I and B100, standard lipid measures, lipid ratios, and CRP as risk factors for cardiovascular disease in women. JAMA. 2005, 294 (3): 326-333. 10.1001/jama.294.3.326. 
16. Kinosian B, Glick H, Garland G: Cholesterol and coronary heart disease: predicting risks by levels and ratios. Ann Intern Med. 1994, 121 (9): 641-647. 10.7326/0003-4819-121-9-199411010-00002.

17. Hsia SH, Pan D, Berookim P, Lee ML: A population-based, cross-sectional comparison of lipid-related indexes for symptoms of atherosclerotic disease. Am J Cardiol. 2006, 98 (8): 1047-1052. 10.1016/j.amjcard.2006.05.024.

18. Barter P, Gotto AM, LaRosa JC, Maroni J, Szarek M, Grundy SM, Kastelein JJ, Bittner V, Fruchart JC, Treating to New Targets I: HDL cholesterol, very low levels of LDL cholesterol, and cardiovascular events. N Engl J Med. 2007, 357 (13): 1301-1310. 10.1056/NEJMoa064278.

19. Ingelsson E, Schaefer EJ, Contois JH, McNamara JR, Sullivan L, Keyes MJ, Pencina MJ, Schoonmaker C, Wilson PW, D'Agostino RB, Vasan RS: Clinical utility of different lipid measures for prediction of coronary heart disease in men and women. JAMA. 2007, 298 (7): 776-785. 10.1001/jama.298.7.776.

20. Kastelein JJ, van der Steeg WA, Holme I, Gaffney M, Cater NB, Barter P, Deedwania P, Olsson AG, Boekholdt SM, Demicco DA, Szarek M, LaRosa JC, Pedersen TR, Grundy SM, TNT Study Group, Ideal Study Group: Lipids, apolipoproteins, and their ratios in relation to cardiovascular events with statin treatment. Circulation. 2008, 117 (23): 3002-3009. 10.1161/CIRCULATIONAHA.107.713438.

21. O'Leary DH, Polak JF, Kronmal RA, Manolio TA, Burke GL, Wolfson SK: Carotid-artery intima and media thickness as a risk factor for myocardial infarction and stroke in older adults. Cardiovascular Health Study Collaborative Research Group. N Engl J Med. 1999, 340 (1): 14-22. 10.1056/NEJM199901073400103. 